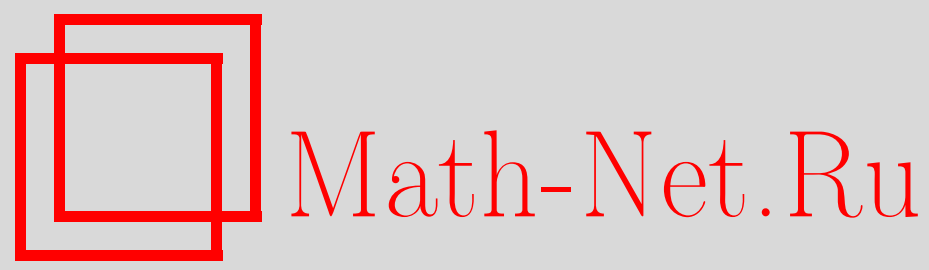

А. П. Буслаев, О методе Лагранжа в нелинейном анализе, Матем. заметки, 1996, том 59, выпуск $3,334-342$

DOI: https://doi.org/10.4213/mzm1721

Использование Общероссийского математического портала MathNet.Ru подразумевает, что вы прочитали и согласны с пользовательским соглашением

http://www . mathnet.ru/rus/agreement

Параметры загрузки:

IP : 3.95 .254 .165

26 апреля 2023 г., 16:30:01

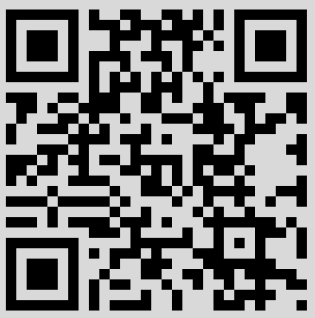




\section{О МЕТОДЕ ЛАГРАНЖА В НЕЛИНЕЙНОМ АНАЛИЗЕ}

\section{А. П. Буслаев}

0. В работе рассматриваются качественные вопросы исследования спектра решений системы

$$
\begin{aligned}
& A^{T}(A x)_{(q)}=\lambda^{q}(x)_{(p)}, A=\left(a_{i j}\right)_{i, j=1}^{m} \\
& \Longleftrightarrow \sum_{i=1}^{m} a_{i j}\left\|\sum_{j=1}^{m} a_{i j} x_{j}\right\|^{q-1} \operatorname{sgn}\left(\sum_{j=1}^{m} a_{i j} x_{j}\right)=\left\|x_{i}\right\|^{p-1} \operatorname{sgn}\left(x_{i}\right)
\end{aligned}
$$

и их связи с поперечниками $d_{n}\left(A B_{p}^{m}, l_{q}^{k}\right) A$-образа $B_{p}^{m}$-эллипсоида для широкого класса квадратных матриц $A, 1 \leqslant p \leqslant \infty$,

$$
\begin{aligned}
B_{p}^{m} & =\left\{x \in \mathbb{R}^{m},\|x\|_{l_{p}^{m}} \leqslant 1\right\}, \\
\partial B_{p}^{m} & =\left\{x \in \mathbb{R}^{m},\|x\|_{l_{p}^{m}}=1\right\} .
\end{aligned}
$$

Эта задача, с одной стороны, является классической задачей спектрального анализа сингулярных чисел $A$ при $p=q=2$ [1], [2], с другой стороны, для вполне положительных матриц (матриц, у которых все миноры всех порядков положительны) исследовалась по параметрам $1<p, q<\infty[3]-[5] . \quad$ При этом использовалась композиция метода Кэллога и теоремы Борсука.

Переход к более широкому классу матриц требует новых подходов. В данной работе в качестве такого подхода предлагается метод Лагранжа, использованньй им при решении одной задачи устойчивости механической системы (колонны) в 1770 году [6].

Работа выполнена при частичной поддержке Российского фонда фундаментальных исследований, грант № 93-01-00237, и Международным научным фондом, грант MP1000. 


\section{1. Обобщенная задача о сингулярных числах. Пусть}

$$
\begin{gathered}
w_{0}, w_{1} \in \mathbb{R}_{+}^{m}, \quad w_{i}=\left\{w_{i k}\right\}_{k=1}^{m}, \\
E_{w_{i}}=\left\{w_{i k} \delta_{k j}\right\}_{k, j=1}^{m}, \quad i=0,1, \quad E=\left\{\delta_{k j}\right\}_{k, j=1}^{m},
\end{gathered}
$$

$A$ - матрица $m \times m, \delta_{k j}$ - символ Кронекера.

Рассмотрим следующую задачу

$$
A^{T} E_{w_{1}}(A x)=\lambda^{2} E_{w_{0}} x,
$$

которая при $w_{0}=w_{1}=(1, \ldots, 1)$ есть задача о сингулярных числах матрицы $A[1],[2]$.

Ясно, что при $w_{0} \in$ int $\mathbb{R}_{+}^{m}$ существует $m$ сингулярных чисел - решений векового уравнения

$$
\left|A^{T} E_{w_{1}} A-\lambda^{2} E_{w_{0}}\right|=0 \Longleftrightarrow\left|A^{T} E_{w_{1}} A E_{w_{0}^{-1}}-\lambda^{2} E\right|=0, w_{0} \in \operatorname{int} \mathbb{R}_{+}^{m},
$$

где $w_{0}^{-1}=\left\{w_{0 k}^{-1}\right\}_{k=1}^{m}$.

Обозначим их в порядке убывания

$$
\lambda_{1}\left(w_{0}, w_{1}\right) \geqslant \lambda_{2}\left(w_{0}, w_{1}\right) \geqslant \cdots \geqslant \lambda_{m}\left(w_{0}, w_{1}\right) \geqslant 0 .
$$

При этом каждому $\lambda_{i}$ соответствует собственньй вектор $X_{i}$, единственньй с точностью до нормировки, если в (3) все неравенства строгие. В силу непрерьвной зависимости нулей многочлена (2) от своих коэффициентов множество $O=\left\{w_{0}, w_{1}\right\} \in \mathbb{R}_{+}^{2 m}$ таких точек (общего положения) открыто.

На этом множестве $O$ определим отображения

$$
\begin{aligned}
& F_{i}:\left(w_{0}, w_{1}\right) \rightarrow \lambda_{i}\left(w_{0}, w_{1}\right) \in \mathbb{R}_{+}, \quad 1 \leqslant i \leqslant m, \\
& G_{i}:\left(w_{0}, w_{1}\right) \rightarrow X_{i}\left(w_{0}, w_{1}\right), \quad\left\|X_{i}\right\|_{l_{2}^{m}}=1, \quad 1 \leqslant i \leqslant m .
\end{aligned}
$$

Легко видеть, что на множестве $O$ отображения $F_{i}, G_{i}$ непрерывны, причем $G_{i}$ - двузначное отображение. Договоримся теперь о том, как определить $F_{i}, G_{i}$ за пределами $O$.

Во-первых, если $w_{0}$ выходит на границу $\mathbb{R}_{+}^{m}$, т.е. некоторые координаты $w_{0 j}, j=j_{1}, j_{2}, \ldots, j_{k}$, стремятся к нулю, то сингулярные числа

$$
\lambda_{1}\left(w_{0}, w_{1}\right), \lambda_{2}\left(w_{0}, w_{1}\right), \ldots, \lambda_{k}\left(w_{0}, w_{1}\right)
$$

в предельной точке $\widehat{w}_{0}$ равны бесконечности, а линейная оболочка соответствующих собственных векторов в пределе переходит в "особое" линейное подпространство

$$
L_{k}=\left\{x=\left(x_{1}, \ldots, x_{m}\right), x_{j}=0, j \neq\left\{j_{1}, \ldots, j_{k}\right\}\right\} .
$$


В этом случае $X_{k}=L_{k} \cap \partial B_{2}^{m}$.

Во-вторьх, если в неравенствах (3) некоторые соотношения суть равенства

$$
\lambda_{i-1}\left(w_{0}, w_{1}\right)>\lambda_{i}\left(w_{0}, w_{1}\right)=\cdots=\lambda_{i+j}\left(w_{0}, w_{1}\right) \geqslant \lambda_{i+j+1}\left(w_{0}, w_{1}\right),
$$

то отображения $G_{k}, i \leqslant k \leqslant i+j$ многозначны

$$
G_{k}:\left(w_{0}, w_{1}\right) \rightarrow x \in \operatorname{lin}\left\{X_{i}, X_{i+1}, \ldots, X_{i+j}\right\} \cap \partial B_{2}^{m} .
$$

В-третьих, если некоторые компоненты $w_{0 j}$ равны бесконечности, то это означает, что в (1) $x_{j}=0$. Если же обрашается в бесконечность $w_{1 j}$, то $(A x)_{j}=0$.

Таким образом, спектральная задача (1) определена для всех наборов весов $w_{0}, w_{1} \in \overline{\mathbb{R}}_{+}^{m}$, кроме тех тривиальных случаев, когда оба вектора имеют все нулевые или бесконечные координаты.

2. Конструкция общего отображения Лагранжа. Пусть $W_{i} \in \overline{\mathbb{R}}_{+}^{m}, i=0,1$ - множества, гомеоморфные $\partial B_{2}^{m} \cap \mathbb{R}_{+}^{m}$. Обозначим через $\left|X_{i}\left(w_{0}, w_{1}\right)\right|$ вектор, координаты которого равны модулям соответствующих координат $X_{i}\left(w_{0}, w_{1}\right)$; при этом отображение $X_{i} \rightarrow\left|X_{i}\right|$ обозначается через $M$.

Далее, пусть $P=\left(P_{0}, P_{1}\right)$ - гомеоморфизм,

$$
\begin{aligned}
& \left(\mathbb{R}_{+}^{m} \cap \partial B_{2}^{m}, \mathbb{R}_{+}^{m} \cap \partial B_{2}^{m}\right) \quad \text { и } \quad B:=\left(W_{0}, W_{1}\right), \\
& P_{0}: \mathbb{R}_{+}^{m} \cap \partial B_{2}^{m} \leftrightarrow W_{0}, \quad P_{1}: \mathbb{R}_{+}^{m} \cap \partial B_{2}^{m} \leftrightarrow W_{1} .
\end{aligned}
$$

Для фиксированного $k, 1 \leqslant k \leqslant m$, определим отображение (вообще говоря, многозначное) $H_{k}=P M G_{k}$, где

$$
\begin{gathered}
G_{k}:\left(w_{0}, w_{1}\right) \in B \rightarrow X_{k}\left(w_{0}, w_{1}\right), \\
M: X_{k}\left(w_{0}, w_{1}\right) \rightarrow\left|X_{k}\left(w_{0}, w_{1}\right)\right|, \\
P:\left|X_{k}\left(w_{0}, w_{1}\right)\right| \rightarrow\left(\widehat{w}_{0}, \widehat{w}_{1}\right) \in B,
\end{gathered}
$$

где под $X_{k}\left(w_{0}, w_{1}\right)$ понимаем нормированный с точностью до знака вектор (4) или множество (5).

Если $X_{k}$ содержит более одного вектора, то оператор $P$ отражает в $\mathbb{R}_{+}^{m}$ каждый элемент $X_{k}$. Непосредственно из определения ясно, что $H_{k}-$ непрерьвное сверху многозначное отображение $B \rightarrow B$ [7, с. 109].

Теорема 1. Для любого $k, 1 \leqslant k \leqslant m$, существует неподвижная точка отображсения $H_{k}$.

Утверждение теоремы получается из следующей конструкции. Далее $X$ - полное метрическое пространство, $d(x, y)$ - расстояние, $F$ - многозначное отображение $X \rightarrow X, F(x) \neq \varnothing \forall x \in X$. Движением называется бесконечная последовательность $\left\{x_{i}\right\}_{i=0}^{\infty}$ такая, что $x_{n+1} \in F\left(x_{n}\right) \forall n$. 
Tеорема A [7, с. 235]. Пусть существует неотрицательная функиия $U: X \rightarrow \mathbb{R} \cup(+\infty)$ такая, что $\forall x \in X, y \in F(x)$

$$
U(y)+d(x, y) \leqslant U(x) .
$$

Тогда для любого $x$ : $U(x)<\infty$ существует движение $x_{n}(x), x_{0}=x$, $x_{n} \rightarrow \bar{x}$. Если график $F$ замкнут, то $\bar{x}-$ неподвижная точка этого отображения.

Теорема Б [7, с. 237]. Пусть $F$ - многозначное отображсение из $X$ в $X$ maкое, что $\forall x \in X$

$$
U_{F}(x):=\inf _{\left\{\left\{x_{n}\right\} \mid x_{0}=x\right.} \sum_{n=0}^{\infty} d\left(x_{n}, x_{n+1}\right) .
$$

Если F полунепрерывно сверху и имеет компактные значения, то $U_{F}$ удовлетворяет (6) $\forall x \in X, y \in F(x)$, а также

$$
U_{F}(x) \geqslant U_{F}(y)+d(x, y) .
$$

Кроме того, еслиU - произвольная функция, удовлетворяющая (6), $m o \forall x \in X$

$$
U_{F}(x) \leqslant U(x)
$$

3. $(p, q)$-спектры. Пусть $p, q$ - вешественные числа, $1<p, q<\infty$,

$$
x=\left(x_{1}, \ldots, x_{m}\right) \in \mathbb{R}^{m}, \quad(x)_{(p)}:=\left(\left|x_{1}\right|^{p-1} \operatorname{sgn} x_{1}, \ldots,\left|x_{m}\right|^{p-1} \operatorname{sgn} x_{m}\right) .
$$

Система уравнений

$$
A^{T}(A x)_{(q)}=\lambda^{q}(x)_{(p)}
$$

при $p=q=2$ является линейной системой для определения сингулярных чисел матрицы $A$, которые упорядочены в соответствии с (3), и при этом каждому числу $\lambda_{i}$ соответствует не менее одного собственного вектора. В том случае, когда

$$
(p-2)^{2}+(q-2)^{2} \neq 0, \quad 1 \leqslant p, q \leqslant \infty,
$$

ее решения исследованы для вполне положительных матриц $A[3]-[5]$.

В частности, в [5] установлено существование спектра $\left(\lambda_{k}, X_{k}\right)$ с $k$ переменами знака координат вектора $X_{k}=\left(X_{k 1}, \ldots, X_{k m}\right)$ для любого $k$, $1 \leqslant k \leqslant m$. При этом

$$
\lambda_{1}>\lambda_{2}>\cdots>\lambda_{k}>\cdots>\lambda_{m}>0 .
$$


Для невырожденных матрищ $A$, которые не являются вполне положительными, из теории Люстерника-Шнирельмана следует лишь сушествование $m$ решений системы (7).

Качественное описание этих решений остается за пределами упомянутой общей теории, поскольку, как отмечалось самим Л.А. Люстерником [8], [9], вычисление категории множества вообще (в частности, линии уровня отношения Релея $\left.\|A x\|_{l_{q}^{m}} /\|x\|_{l_{p}^{m}}\right)$ является очень трудной задачей.

Перейдем к конкретизации метода Лагранжа. Пусть

$$
\begin{gathered}
1<p, q<\infty, \quad p \neq 2, \quad q \neq 2, \\
s=s(p)=\frac{p}{p-2}, \quad r=r(q)=\frac{q}{q-2}, \\
W_{0}=\left\{w_{0} \in \mathbb{R}_{+}^{m},\left\|w_{0}\right\|_{l_{s}^{m}}=1\right\}, \\
W_{1}=\left\{w_{1} \in \mathbb{R}_{+}^{m},\left\|w_{1}\right\|_{l_{r}^{m}}=1\right\},
\end{gathered}
$$

$A$ - невырожденная квадратная матрица, $B=\left(W_{0}, W_{1}\right)$. Каждой паре $\left(w_{0}, w_{1}\right) \in B$ сопоставим спектральную пару (1)

$$
\left(\lambda_{k}\left(w_{0}, w_{1}\right), X_{k}\left(w_{0}, w_{1}\right)\right),
$$

где $X_{k}$ - собственное подпространство, пересеченное с $\partial B_{2}^{m}$.

Каждьй вектор $x_{k}$ из $X_{k}$ отображается в $\left(w_{0}, w_{1}\right)$ следующим образом

$$
\left\{\begin{array}{l}
\bar{w}_{0}=\gamma_{0}\left|x_{k}\right|^{p-2}, \\
\bar{w}_{1}=\gamma_{1}\left|A x_{k}\right|^{q-2},
\end{array}\right.
$$

где $\gamma_{0}$ и $\gamma_{1}-$ нормировочные константы:

$$
\begin{aligned}
\left\|\bar{w}_{0}\right\|_{s} & =\left\|\bar{w}_{0}\right\|_{p /(p-2)}=\gamma_{0}\left\|x_{k}\right\|_{p}^{p-2}=1, \\
\left\|\bar{w}_{1}\right\|_{r} & =\left\|\bar{w}_{1}\right\|_{q /(q-2)}=\gamma_{1}\left\|A x_{k}\right\|_{q}^{q-2}=1 .
\end{aligned}
$$

Согласно теореме 1 существует неподвижная точка построенного отображения $(p>2, q>2)$

$$
w_{0}^{k}=\bar{w}_{0}^{k}, \quad w_{1}^{k}=\bar{w}_{1}^{k},
$$

т.е.

$$
\begin{aligned}
w_{0}^{k}=\gamma_{0}\left|x_{k}\right|^{p-2}, w_{1}^{k}=\gamma_{1}\left|A x_{k}\right|^{q-2} & \Longleftrightarrow\left(A^{T} E_{w_{1}^{k}}\left(A x_{k}\right)=\lambda^{2} E_{w_{0}^{k}} x_{k}\right) \\
& \Longleftrightarrow A^{T}\left(A x_{k}\right)_{(q)}=\lambda_{k}^{q}\left(x_{k}\right)_{(p)} .
\end{aligned}
$$


Если же $p<2$ или (и) $q<2$, то построенное отображение дополним радиальной проекцией $W^{\prime} \leftrightarrow W_{0}, W_{1} \leftrightarrow W^{\prime}$, где $W^{\prime}=\left\{\omega \in \mathbb{R}_{+}^{m},\|\omega\|_{l_{2}^{m}}=1\right\}$, и далее аналогично.

Обозначим через

$$
\Lambda_{k}=\Lambda_{k}(A, p, q)
$$

множество тех $\lambda \geqslant 0$, для которых

а) система (7) имеет нетривиальное решение $x \neq 0$, т.е. $(\lambda, x)-$ спектральная пара,

б) вектор $x \in X_{k}\left(w_{0}, w_{1},\right)$, где

$$
w_{1}=\gamma_{1}|A x|^{q-2}, \quad w_{0}=\gamma_{0}|x|^{p-2},
$$

и константы $\gamma_{1}>0, \gamma_{0}>0$ выбраны таким образом, чтобы

$$
w_{1} \in \partial B_{(q / 2)^{\prime}}^{m}, \quad w_{0} \in \partial B_{(p / 2)^{\prime}}^{m},
$$

т.e. $\left(a^{-1}+a^{\prime-1}\right)=1$,

$$
\left\|w_{1}\right\|_{l_{q /(q-2)}^{m}}=1, \quad\left\|w_{0}\right\|_{p /(p-2)}^{m}=1
$$

Теорема 2. Множество нетривиальных решений системы (7) непусто для любой квадратной матрицы $A \neq 0$. Если каждое решение $\left(\lambda^{*}, x^{*}\right)(7)$ неособое, m.e.

$$
\left.\left(A^{T}(A x)_{(q)}-\lambda(x)_{(p)}\right)\right|_{x=x^{*}, \lambda=\lambda^{*}}
$$

невирождена, то для любого $k, 1 \leqslant k \leqslant m, X_{k} \neq 0 u \Lambda_{k} \cap \Lambda_{j}=\varnothing$ $\forall k, j, k \neq j$.

ДокАЗАТЕЛЬСтво. Непустота множества нетривиальных решений (7) следует из того, что в экстремальной задаче

$$
\frac{\|A x\|_{l_{q}^{m}}}{\|x\|_{l_{p}^{m}}} \rightarrow \max ,
$$

максимум достигается на решении (7).

В силу теоремы 1 каждое множество $\Lambda_{k}, 1 \leqslant k \leqslant m$, непусто. Из-за условия теоремы 2

$$
X_{k} \cap X_{j}=\varnothing .
$$

Следовательно, для любого $k(7)$ имеет решение $x^{(k)} \in X_{k}$ и

$$
x^{(k)} \notin X_{j} \quad \forall j \neq k \text {. }
$$


4. Метод Лагранжа. Рассмотрим следующий класс задач на экстремум

$$
\Lambda_{k}\left(w_{0}, w_{1}\right) \stackrel{\left(w_{0}, w_{1}\right)}{\rightarrow} \operatorname{extr}
$$

где

$$
\left\|w_{0}\right\|_{p /(p-2)}^{m}=\left\|w_{1}\right\|_{l_{q /(q-2)}^{m}}=1, \quad\left(w_{0}, w_{1}\right) \subset M_{1} \subset \mathbb{R}_{+}^{2 m},
$$

$M_{1}$ - некоторое подмножество.

Бесконечномерные частные случаи задачи (8), (9), когда $A$ - оператор интегрирования, а $x$ - вещественнозначная функция, определенная на отрезке, исследовались в огромном числе работ. Большая часть этих исследований связана с механическими приложениями и относится к старшим точкам спектра $(k=1 \vee 2)$. Отметим среди них работы [10]-[15].

Пусть

$$
B_{l_{p\left(w_{0}\right)}^{m}}^{m}=\left\{x \in \mathbb{R}^{m},\|x\|_{l_{p\left(w_{0}\right)}^{m}} \leqslant 1\right\}, \quad\|x\|_{l_{p\left(w_{0}\right)}^{m}}=\left(\sum_{i=1}^{m} x_{i}^{p} w_{0} i\right)^{1 / p} .
$$

Хорошо известно [3], [4], что колмогоровский поперечник $A B_{2\left(w_{0}\right)}^{m}$ в $l_{2\left(w_{1}\right)}^{m}$ совпадает со спектром $\lambda_{k}\left(w_{0}, w_{1}\right)$

$$
d_{k}\left(A B_{2\left(w_{0}\right)}^{m}, l_{2\left(w_{1}\right)}^{m}\right)=\lambda_{k}\left(w_{0}, w_{1}\right),
$$

где

$$
d_{k}\left(A B_{p\left(w_{1}\right)}^{m}, l_{q\left(w_{0}\right)}^{m}\right)=\min _{L_{k-1}} \sup _{x} \min _{y \in L_{k-1}} \frac{\|A x-y\|_{l_{q\left(w_{1}\right)}}}{\|x\|_{l_{p\left(w_{0}\right)}}} .
$$

Предположим, что $q>2, p<2$. Оценим

$$
\sup _{\left\|w_{0}\right\|_{l(p)}} \sup _{\substack{l \\ s(p)}} \lambda_{k}\left(w_{l}, w_{0}, w_{1}\right) .
$$

В силу (10)

$$
\begin{aligned}
& \sup _{\left\|w_{0}\right\|_{l_{s(p)}^{m}}=1} \sup _{\left\|w_{1}\right\|_{l_{r(q)}^{m}}=1} \lambda_{k}\left(w_{0}, w_{1}\right) \\
&=\operatorname{lw}_{\left\|w_{0}\right\|_{l(p)}} \sup _{s(p)}=w_{1} \|_{l_{r(q)}^{m}}=1 \\
& \min _{L_{k-1}} \sup _{x} \min _{y \in L_{k-1}} \frac{\|A x-y\|_{l_{2\left(w_{1}\right)}}}{\|x\|_{l_{2\left(w_{0}\right)}}} \\
& \leqslant \min _{L_{k-1}} \sup _{x} \min _{y \in L_{k-1}} \frac{\sup _{\left\|w_{1}\right\|_{l_{r(q)}^{m}}^{m}=1}\|A x-y\|_{l_{2\left(w_{1}\right)}}}{\sup _{\left\|w_{0}\right\|_{l_{s(p)}^{m}}=1}\|x\|_{l_{2\left(w_{0}\right)}}} \\
&=\min _{L_{k-1}} \sup _{x} \min _{y \in L_{k-1}} \frac{\|A x-y\|_{l_{q}}}{\|x\|_{l_{p}}}=d_{k}\left(A B_{p}^{m}, l_{q}^{m}\right) .
\end{aligned}
$$


В случае $q<2, p>2$ аналогичные рассуждения приводят к неравенствy

$$
\left.\left\|w_{0}\right\|_{l_{s(p)}^{m}} \inf _{\left\|w_{1}\right\|_{l} m=1} \lambda_{r(q)}\left(w_{0}, w_{1}\right)\right] \geqslant d_{k}\left(A B_{p}^{m}, l_{q}^{m}\right) .
$$

Таким образом, имеет место

ТЕОРема 3. Для любой квадратной матрицы $A, 1 \leqslant k \leqslant m$, имеют место следующие оченки $\Lambda_{k}(A, p, q)$ :

а) если $\infty>q>2>p>1$, mo

$$
\left(\sup \lambda, \lambda \in \Lambda_{k}(A, p, q)\right) \leqslant d_{k}\left(A B_{p}^{m}, l_{q}^{m}\right) ;
$$

б) если $1<q<2<p<\infty$, то

$$
\left(\inf \lambda, \lambda \in \Lambda_{k}(A, p, q)\right) \geqslant d_{k}\left(A B_{p}^{m}, l_{q}^{m}\right) .
$$

Остановимся теперь на анализе неравенства (11).

Теорема 4. В условиях теоремы 3б) и в предположсении о невырожденности $\check{\lambda}_{k}(A, p, q)$ - минимального собственного значения из $\Lambda_{k}(A, p, q)$,

$$
\check{\lambda}_{k}(A, p, q)=\operatorname{iw}_{0} \|_{l_{s(p)}^{m}} \inf _{=\left\|w_{1}\right\|_{l} l_{r(q)}^{m}=1} \lambda_{k}\left(A, l_{2\left(w_{1}\right)}^{m}, l_{2\left(w_{0}\right)}^{m}\right)=d_{k}\left(A B_{p}^{m}, l_{q}^{m}\right) .
$$

ДокАЗАТЕЛЬСтво. Первое равенство следует из того, что функция $\lambda_{k}\left(A, l_{2\left(w_{1}\right)}^{m}, l_{2\left(w_{0}\right)}^{m}\right)$ непрерьвна на ограниченном множестве.

Для любого линейного пространства размерности $k L_{k} \in \mathbb{R}_{+}^{m}$, найдутся

$$
w_{1}, w_{0} \in \mathbb{R}_{+}^{m}, \quad w_{0} \in \partial B_{r(q)}^{m}, w_{1} \in \partial B_{s(p)}^{m}
$$

такие, что

$$
L_{k}=\operatorname{lin}\left\{x_{2\left(w_{1}\right) 2\left(w_{0}\right)}^{(1)}, \ldots, x_{2\left(w_{1}\right) 2\left(w_{0}\right)}^{(k)}\right\},
$$

где $\left(\lambda_{i\left(2\left(w_{1}\right) 2\left(w_{0}\right)\right)}, x_{2\left(w_{1}\right) 2\left(w_{0}\right)}^{(i)}\right)$ - спектральные пары, $1 \leqslant i \leqslant k$.

Рассмотрим экстремальную задачу

$$
\lim _{s \rightarrow+\infty} \min _{x \in L_{k}} \frac{\|A z(s)\|_{l_{q}}}{\|z(s)\|_{l_{p}}},
$$

где $z(s)$ - последовательность векторов, определенная по системе $s=1,2, \ldots, z(0)=x$,

$$
A^{T}(A z(s-1))_{\left(2\left(w_{0}\right)\right)}=\mu_{s}(z(s))_{\left(2\left(w_{0}\right)\right)},
$$

$\mu_{s}$ определяется из условия нормировки. 
При фиксированном $x$

$$
\lim _{s \rightarrow+\infty} \frac{\|A z(s)\|_{l_{q}}}{\|z(s)\|_{l_{p}}}
$$

сходится со скоростью геометрической прогрессии. Поэтому значение задачи (12) равно

$$
\begin{aligned}
\min _{1 \leqslant l \leqslant k} \frac{\left\|A x^{(l)}\right\|_{2\left(w_{1}\right) 2\left(w_{0}\right)}}{\left\|x_{2\left(w_{1}\right) 2\left(w_{0}\right)}^{(l)}\right\|_{p}} & \geqslant \min _{1 \leqslant l \leqslant m} \frac{\left\|A x^{(l)}\right\|_{2\left(w_{1}\right)}\left\|w_{1}\right\|_{r(q)}}{\left\|x^{(l)}\right\|_{2\left(w_{0}\right)}\left\|w_{0}\right\|_{s(p)}} \\
& =\min _{1 \leqslant l \leqslant k} \lambda_{l}\left(2\left(w_{1}\right) 2\left(w_{0}\right)\right)=\lambda_{k}\left(2\left(w_{1}\right) 2\left(w_{0}\right)\right) .
\end{aligned}
$$

Поскольку (12) оценивает колмогоровский поперечник снизу [5], то получаем неравенство, обратное (11).

\section{СПИСОК ЦИТИРОВАННОЙ ЛИТЕРАТУРЫ}

[1] Гантмахер Ф.Р. Теория матриц. М.: Наука, 1987.

[2] Гантмахер Ф. Р., Крейн М. Г. Осцилляционные матрицы, ядра и малые колебания механических систем. М.: Гостехиздат, 1953.

[3] Тихомиров В. М. Итоги науки и техн. Соврем. пробл. матем. Фундамент. направления. Т. 14. М.: ВИНИТИ, 1987.

[4] Pinkus A. Some extremal problems for strictly totally positive matrices // Linear Algebra and its Appl. 1985. V. 64. P. 141-156.

[5] Буслаев А. П. О вариационном описании спектра вполне положительных матриц и экстремальных задачах теории приближений // Матем. заметки. 1990. T. 47. № 1. C. 39-46.

[6] Lagrange G. L. Eurves. V. 2. Paris: Gauthier-Villars, 1868.

[7] Обен Ж. П., Экланд И. Прикладной нелинейный анализ. М.: Мир, 1988.

[8] Люстерник Л. А. Топологические основы общей теории собственных значений // УМН. 1970. Т. 25. №4(154).

[9] Люстерник Л.А. Некоторые вопросы нелинейного функционального анализа // УМН 1956. Т. 11. №6 (72). С. 145-168.

[10] Сейранян А. П. Об одной задаче Лагранжа // Изв. АН СССР. Сер. матем. 1984. T. 48. № 2. C. 101-111.

[11] Ольхофф Н. Оптимальное проектирование конструкций. М.: Мир, 1981.

[12] Егоров Ю. В., Кондратьев В.А. Об оценках первого собственного значения задачи Штурма-Лиувилля // УМН. 1984. Т. 39. №2 2(236). С. 151-152.

[13] Keller J. The shape of the strongest column // Arch. Rat. Mech. Anal. 1960. № 5. P. $275-285$.

[14] Tadjbaksh I., Keller J. Strongest columns and isoperimetric inequalities for eigenvalues // J. Appl. Mech. 1962. V. 29. P. 159-164.

[15] Cox S. J. The Shape of the Ideal Column // The Mathematical Intelligenger. 1992. V. 14. № 1. P. 16-24. 